\title{
Probiotic Supplementation and Gastrointestinal Endotoxemia Before and After the Marathon Des Sables.
}

Craig A. Suckling ${ }^{1}$, Justin D. Roberts ${ }^{1}$, Georgia Y. Peedle ${ }^{1}$, Dan A. Gordon ${ }^{1}$, Hannah Marshall $^{2}$, Lee Taylor ${ }^{2}$ and Michael G. Roberts ${ }^{3}$. Anglia Ruskin University, United Kingdom ${ }^{1}$; University of Bedfordshire, United Kingdom ${ }^{2}$; University of Hertfordshire, United Kingdom ${ }^{3}$.

Whilst evidence of increased gastrointestinal endotoxemia (GE) has been previously demonstrated during single-day ultra-endurance events, less is known on the prevalence of GE following extreme ultra-events such as the Marathon Des Sables (MDS). The potential benefit of probiotic formulas on gut integrity during ultraendurance events also requires further investigation.

PURPOSE: To assess the impact of probiotic supplementation with or without glutamine on GE prevalence in runners competing in a multi-day ultra-run (MDS).

METHODS: Thirty four healthy participants from the 2015 MDS UK cohort volunteered for a 12 week pre-race intervention and were randomly assigned to either: probiotic (PRO; 100mg.d $\mathrm{d}^{-1}$ lactobacillus acidophilus) （age $40 \pm 3$ yrs., weight 79.4 $\pm 2.0 \mathrm{~kg}, \mathrm{VO}_{2 \max } 4.2 \pm 0.1$ L.min ${ }^{-1}$ ), probiotic with glutamine $\left(\mathrm{PRO}_{\text {glut }} ; 40.5 \mathrm{mg} . \mathrm{d}^{-1}\right.$

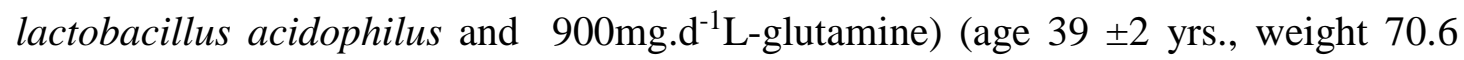

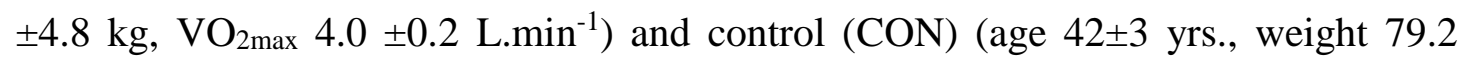

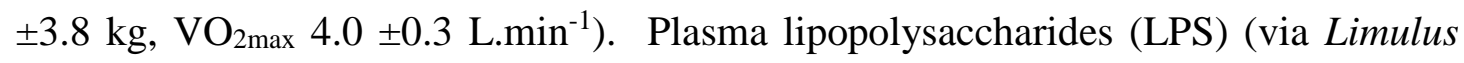
Amebocyte Lysate chromogenic endotoxin quantification) were assessed at weeks 0 , 12, post-race and 7 days post-race. Performance data was collated from official timing chips. Data presented as mean \pm SE.

RESULTS: Mild to moderate GE was prevalent in all groups at baseline (PRO 9.71 \pm 0.85 pg. $\mathrm{ml}^{-1}, \mathrm{PRO}_{\text {glut }} 9.89 \pm 1.43$ pg.ml ${ }^{-1}, \mathrm{CON} 9.40 \pm 0.57$ pg.ml $\mathrm{ml}^{-1}$; P>0.05). Whilst LPS, post intervention, was lower in $\mathrm{PRO}_{\text {glut }}$ there was no significance between groups $\left(9.81 \pm 1.47\right.$ pg.ml ${ }^{-1}$ vs $12.80 \pm 0.93$ pg.ml ${ }^{-1}$ (PRO) vs $11.72 \pm 1.08$ pg. $\mathrm{mol}^{-1}$ $(\mathrm{CON}) ; \mathrm{P}>0.05)$. LPS were evidently reduced $6 \mathrm{hrs}$ post-race, but not different between groups (PRO: $7.29 \pm 1.41$ pg. $\mathrm{ml}^{-1}$, PRO $_{\text {glut: }} 6.95 \pm 0.94$ pg.ml ${ }^{-1}, \mathrm{CON}: 9.73$ 
\pm 1.39 pg. $\mathrm{ml}^{-1} ; \mathrm{P}>0.05$ ).Plasma LPS returned to baseline levels 7 days post-race (PRO $7.60 \pm 0.95$ pg.ml ${ }^{-1}, \mathrm{PRO}_{\text {glut }} 10.41 \pm 1.04$ pg. $\mathrm{ml}^{-1}, \mathrm{CON} 8.57 \pm 0.75$ pg.ml $\left.{ }^{-1} ; \mathrm{P}>0.05\right)$. Race performance (hrs:mins) was not significant between groups, despite PRO and

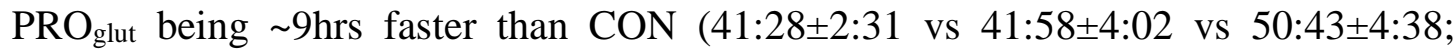
$\mathrm{P}>0.05)$.

CONCLUSION: Moderate GE was prevalent in all groups pre-race and fell significantly during the short-term recovery period. Despite promising results neither probiotic formula had a significant impact on GE or race performance. 